\title{
El patrimonio granadino como elemento didáctico: un itinerario inclusivo por el Barrio de La Manigua
}

\author{
Salvador Mateo Arias Romero ${ }^{1}$ \\ Javier Contreras García \\ María de Czestochowa Molina Serrano ${ }^{3}$ \\ Javier Tenedor Tenedor ${ }^{4}$
}

Recibido 05 agosto de 2019 · Aceptado 30 de octubre de 2019

\section{RESUMEN}

Esta propuesta tiene la intención de poner en valor un tradicional barrio granadino como es el de La Manigua, barrio cuyo nombre pasa desapercibido para la mayoría de los granadinos. Las intervenciones llevadas a cabo sobre sus edificaciones y trazado cambiaron completamente la fisonomía del espacio allá por fines de los años treinta, alargándose las obras hasta finales de los cuarenta. El resultado fue la construcción de un barrio céntrico de alto valor simbólico para la arquitectura del momento y de revitalización del espacio, así como de saneamiento, al tiempo que transformaba su tradicional carácter negativo en una de las zonas más elitistas de la ciudad, tanto económica como culturalmente

Palabras clave: didáctica, patrimonio, granada; inclusivo.

Granada's Heritage as a Didactic Element: An Inclusive Tour through the La Manigua Neighborhood

\section{ABSTRACT}

This proposal intends to highlight the value of a traditional Granada neighborhood, like that of La Manigua, whose name is unknown to most Granada residents. The interventions carried out with respect to the constructions and layout of the neighborhood completely changed to physiognomy of the space between the late 1930s and the late 1940s. The result was the construction of a centric neighborhood with high symbolic value for architecture at the time and the revitalization and beautification of the area, transforming its traditionally negative nature to become one of the city's most elitist areas, in terms of both economy and culture.

Key words: Didactics, heritage, Granada, inclusive.

I Doctor. Profesor ayudante, Departamento de Didáctica de las Ciencias Sociales, Facultad de Ciencias de la Educación, Universidad de Granada. Contacto: mateoarias@ugr.es

2 Profesor sustituto interino, Departamento de Didáctica de las Ciencias Sociales, Facultad de Ciencias de la Educación, Universidad de Granada. Contacto: jcontreras@ugr.es

3 Grupo de Investigación Patrimonio Arquitectónico y Urbano en Andalucía, Departamento de Historia del Arte, Universidad de Granada, Fundación Patronato Avemariano de Granada. Contacto: czestochowa@gmail.com

4 Profesor ayudante, Universidad de Granada. Contacto: javiertenedor@correo.ugr.es 


\section{Introducción}

Nuestra propuesta, basada en un itinerario cultural por el barrio que albergaría importantes centros de la vida cultural granadina como el mítico Teatro Cervantes (ya desaparecido), y en la actualidad el Teatro Isabel la Católica o el Centro Artístico, supone un acercamiento a ideas de carácter inclusivo, basadas en propuestas de integración para personas con diversidad, usando las Tecnologías de la Información y Comunicación (TIC).

Tras reconocer los valores patrimoniales, culturales e históricoartísticos de este barrio granadino, incluimos en este Itinerario cultural una propuesta de actividades, así como un plan de trabajo concretado para englobar la inclusión, las TIC junto a la inteligencia emocional, siendo esta última esencial y la premisa indispensable a la hora de llevar a cabo cualquier itinerario cultural.

De nada serviría la existencia del patrimonio histórico-artístico si, desde la educación como herramienta de transformación social, no se permitiera al alumnado aprender a conocer el patrimonio, contemplarlo, valorarlo y disfrutarlo, para trasvasar ese conocimiento a sus familiares $y$, por ende, a las generaciones del futuro.

El mundo en que vivimos y la sociedad de las tecnologías y el conocimiento en la que nos encontramos, nos invitan a desarrollar nuestra labor docente aplicando a los métodos tradicionales de enseñanza (clase magistral, libros de texto, cuadernos, bolígrafos, lápiz, etc.), nuevas herramientas como las TIC. Asimismo, hay que tener en cuenta que, si nuestro alumnado no posee unas mínimas nociones básicas por parte del docente sobre el barrio de La Manigua, posteriormente no podrá llevar a cabo esta actividad, siendo realmente el alumnado protagonista de esta experiencia de enseñanza-aprendizaje.

\section{Contexto histórico: Granada en los años 30}

La Granada de principios de la década de los años treinta distaba mucho de parecerse a la ciudad que conocemos hoy. Gran parte de esta diferenciación se basa en la transformación llevada a cabo en el 
centro de la ciudad, en el conocido barrio de La Manigua, durante el periodo en la alcaldía de Antonio Gallego Burín, catedrático de Historia del Arte de la Universidad de Granada y figura fundamental de la vida granadina en los años del primer franquismo.

Hay que tener en cuenta dos hitos de la transformación de la ciudad, como serán el Embovedado y la apertura de la Gran Vía de Colón, lo que supondrá un empuje a las reformas urbanas granadinas que durante siglos habían estado en franca decadencia.

Como han señalado otros autores, como Julio Juste o Ángel Isac, Granada careció de cualquier cosa parecida a un plan de alineaciones hasta los proyectos de Gallego Burín, de I943 y I95I, si bien conocemos la existencia de otras leyes nacionales y de una serie de ordenanzas municipales, (constantemente violadas), que deberían haber marcado el devenir de las construcciones en la ciudad.

Por tanto, en nuestro caso, serán de vital importancia todos los proyectos de reforma interior, desde el de 1922 hasta el aprobado en 1938, obra de Francisco Robles, sin olvidar, claro está, el "mítico" plan de reforma interior y ensanche de 1935. De todas formas, serán los diseños finales de Miguel Olmedo Collantes, sobre la base del proyecto de Francisco Robles, los que se impongan como obras realizadas, al formar este arquitecto un tándem único con el ideólogo de la "nueva Granada", Antonio Gallego Burín.

Lamentamos la falta de espacio en esta aportación para realizar un estudio detallado de la reforma de este céntrico espacio de la ciudad, por lo cual remitimos a la bibliografía específica del tema, para aquellos interesados. No obstante, lo importante de esta transformación reside en cómo se tornó un barrio degradado, en una zona centro basada en los postulados del reformismo haussmaniano, dando como resultado un espacio icónico lleno de edificios representativos y de centros culturales y de poder.

\section{Un itinerario didáctico: la puesta en valor del patrimonio local}

Estos edificios representativos serán por tanto la base de nuestro itinerario didáctico, que aboga por poner en valor nuestro patrimonio 
más cercano, fuera de los típicos e imponentes monumentos que posee nuestra ciudad y que suelen acaparar las intervenciones patrimoniales. Por tanto, será nuestro itinerario el comprendido desde: La Plaza del Carmen, donde destaca la Casa del Ayuntamiento, siguiendo hasta Puerta Real, espacio en el que resaltaremos edificios de la índole de la Casa de Telecomunicaciones y Correos, y en menor medida el Edificio Olmedo y el Hotel Victoria, pasando por la icónica calle Ángel Ganivet, con sus características aceras porticadas e imponentes edificios, estudiando por último el Teatro Isabel la Católica y finalizando nuestra ruta en la Plaza de Mariana Pineda, lugar anexo a donde se encontraba el ya desaparecido Teatro Cervantes.

El hecho de seleccionar este itinerario supone, como decíamos, una puesta en valor de un espacio que, normalmente, pasa desapercibido a los estudios patrimoniales. Poner en relevancia estos edificios nos acerca a un mayor conocimiento de los procesos y desarrollos que se dieron en la transformación urbana más reciente, al tiempo que fomenta un conocimiento más profundo de nuestra historia.

\section{Plaza del Carmen}

La Plaza del Carmen tendrá su origen en los procesos desamortizadores del siglo XIX. En el plano de Granada de Ramón González Sevilla y Juan de Dios Bertuchi de I894, ya encontramos la Plaza como tal, después de que, como decíamos, los procesos de desamortización acabaran con su Claustro, para dar una nueva plaza a la ciudad. Lo importante de este espacio, considerado como una plaza menor por el propio Gallego Burín, y que pasaba por desapercibida para el granadino de a pie, es que alberga la Casa del Ayuntamiento, sede del poder local que ocupa el espacio que, con anterioridad, poseía el antiguo Convento del Carmen. Desde su conversión a nueva Casa Consistorial, hasta las propuestas de reforma de Francisco Prieto Moreno, o las más recientes intervenciones de los años noventa, la Plaza - y en concreto el edificio del Ayuntamiento- se han convertido en espacio principal de la vida granadina.

Proyectos importantes para la valorización es esta plaza llegarán en los años cuarenta, aunque ninguno de ellos verá la luz. Así, 
Francisco Prieto Moreno firmará en 1943 el Proyecto de Reforma de la Casa del Ayuntamiento, con barroca composición, torre incluida, que invocaba en su señorial arquitectura la tradición de las Casas Consistoriales castellanas del siglo de oro. Propuesta similar haría Damián Villar para ornamentar la plaza en I946, con su Monumento a los héroes granadinos de la independencia, monolito decorativo que ocuparía el espacio central de la plaza, con las efigies de cuatro importantes militares de la guerra contra los franceses. En su propuesta, con fuente incluida, se buscaba destacar este espacio del que el propio Gallego Burín diría: siempre los granadinos pasan por ella sin mirar siquiera esta plaza.

\section{Puerta Real}

La idea de convertir el espacio de la Puerta Real en una especie de rotonda o plaza circular, parte del proyecto de reforma de Ángel Casas de 1929. Esta idea se consolidará en todos los proyectos posteriores, de forma que se asumirá este espacio circular como modelo de la misma, si bien llegó a funcionar como rotonda durante mucho tiempo, esta funcionalidad no se adaptó bien al espacio, así que finalmente quedó descartada como tal, dando su configuración actual como la conocemos hoy día.

Los edificios más representativos de este espacio —y que incluimos en nuestro itinerario- serían la Casa de Telecomunicaciones y Correos, el Edificio Olmedo y el Gran Hotel Victoria.

Entre todos ellos destaca por representativo el Palacio de Telecomunicaciones, obra de los arquitectos Otamendi y Lozano Losilla, siendo resultado de una azarosísima vida hasta llegar a su construcción final.

\section{Casa de Correos y Telecomunicaciones}

Para la fecha, Granada contaba con una Casa de Correos que había quedado algo desfasada, situada en espacios cercanos a lo que hoy es la Plaza Isabel la Católica. Desde que en 1934, cuando el Ministerio de Comunicaciones inicie los trámites para el nuevo edificio, hasta 
I95I, cuando este quede finalizado, se propondrán varios espacios y se sucederán varios litigios y controversias, hasta que finamente se superen todas ellas, dándonos como resultado el imponente edificio que conocemos hoy.

$\mathrm{Su}$ arquitectura, eminentemente racionalista, pero basada en modelos de corte clásico, dota de señorialidad a un espacio representativo, como debía ser una Casa de Correos. Su principal ubicación en la reforma de tan céntrico barrio, con fachada a Puerta Real, y su inspiración en los palacios renacentistas italianos ponen en relieve uno de los edificios más importantes de la Granada de los años cuarenta, y también de toda España. El I 5 de julio de 1958 dará comienzo el servicio postal en esta nueva localización.

Otro de los edificios importantes que enmarcan Puerta Real será el Edificio Olmedo. Ideado (y comprado en subasta con anterioridad por Miguel Olmedo Collantes) será el I I de agosto de 1943 cuando el Ayuntamiento dé luz verde a las obras. Su estilo, parecido al edificio de Correos, pero de corte menos monumental, se basa en los modelos de la época, tradicionalismo tamizado por el racionalismo de la arquitectura contemporánea. Destaca entre todo el conjunto el cuerpo que remata el edificio, de clara inspiración escurialense.

Otro de los edificios significativos será el Gran Hotel Victoria, cuya arquitectura historicista, con característica cúpula, casi es borrada para siempre con la propuesta de reforma de Miguel Olmedo, quien propuso redefinir las fachadas con fuertes líneas rectas e incorporando una torreta rematada en frontón.

\section{Calle Ángel Ganivet}

La icónica calle Ganivet, una ampliación del trazado original de la vía, con más imagen que practicidad, será la efigie de una reforma que nunca llegó a cumplirse. Las ideas iniciales formaban un circuito de reforma interior que abarcaba la conexión del centro histórico con lo que por aquel entonces eran las zonas de expansión. La calle Ganivet, como primera vía de esta reforma, quedará inconexa por tanto y aislada de este circuito de reforma, lo que la convertirá en un 
ente poco práctico en el circuito viario granadino. Su larga historia y lo significativo de sus edificios, no pueden ser abordados aquí, con lo cual volvemos a remitir a las obras que la han estudiado. Aunque sí podemos destacar sus icónicas galerías porticadas, incluidas en una reforma del proyecto inicial de Francisco Robles el I I de diciembre de 1942, cuando se decida esta adición a la por entonces calle "A" de La Manigua, como alusión a las plazas porticadas castellanas, imagen de la España imperial.

Pasamos ahora por tanto a resaltar dos edificios principales de la cultura granadina, uno ya desaparecido, pero de especial relevancia histórica, como fue el Teatro Cervantes, situado en la plaza del Campillo, en las postrimerías de lo que hoy sería la calle Ganivet, y el nuevo Teatro Isabel la Católica, heredero (solo en nombre) del original situado en la Plaza de los Campos y desaparecido en los actos revolucionarios ocurridos en tiempos de la II República.

\section{Teatro Cervantes}

Según narra Antonio Gallego y Burín, fue el ingeniero Joaquín Pery quien comenzó la construcción del Teatro Cervantes en I802, a iniciativa del Capitán General Vasco. En I804, estando casi finalizado, fue suspendida su obra, convirtiéndose en almacenes militares, hasta que el general francés Sebastiani impulsó de nuevo su terminación. Su inauguración se produjo el I5 de noviembre de I8I0, con el nombre de Teatro Napoleón, cambiado posteriormente por el de Principal y, más tarde, por el de Cervantes, al celebrarse el tercer centenario de la publicación del Quijote.

Por aquellos días, Granada carecía de un teatro digno y únicamente los aficionados al arte escénico poseían algunos míseros "corrales", al estilo de los siglos XVI y XVIII, para representar los autos sacramentales y alguna que otra obra del repertorio clásico. Así es que el general francés encargó la traza y ejecución de la obra con el beneplácito del rey José Bonaparte. Tras su inauguración, el general Sebastiani concurría asiduamente, entrando por la puerta reservada al palco I de la derecha, cuyo uso y dominio disfrutaron los que le sucedieron desde entonces en el mando militar en la ciudad. 
Se marcharon los franceses y, como no se podían llevar el teatro por ellos construido, el Estado se incautó de él y decidió venderlo, para que se mantuviera en buen estado y fuese lugar de divertimento, así como templo del arte escénico.

En I903 aparecía la siguiente noticia en la Revista Albambra sobre el Teatro Cervantes y el cine:

Ya estamos otra vez, a Dios gracias, tranquilos y satisfechos, llenando el Teatro Principal para ver el Bioscope (cinematógrafo, para más claridad), y contemplar extasiados los arlequinadas de Un viaje a la luna, y las prodigiosas vistas tomadas al natural. $(\mathrm{s} / \mathrm{p})$.

Este anuncio no hacía más que confirmar que, desde los inicios del cine, se venían proyectando películas en el teatro Cervantes.

El I de diciembre de 19I2, y cumpliendo con lo acordado en la Junta de Espectáculos, los señores Méndez Vellido, Cendoya y Wilhelmi realizaron una inspección en el Teatro Cervantes, en la que reconocieron todo el inmueble. De dicha inspección resultó que en planta baja existían ocho puertas, que comunicaban directamente con la calle. Los palcos contaban con dos escaleras independientes del resto de las localidades y tres salidas al Liceo. En la entrada general había dos salidas y otra que comunicaba con los palcos. El paraíso constaba de dos escaleras independientes y otra que comunicaba con la entrada general. Al escenario daban salida tres puertas, una de ellas al Café Inglés. En caso de incendios, estaba provisto de forma correcta. El escenario, además de un telón aislador metálico, disponía de otro de aguas y dos potentes mangas de riego, así como en las plateas y palcos. El Teatro Cervantes, podrían desalojarlo en caso de alarma en unos tres minutos, utilizando las veintitrés puertas que tiene dispuestas.

Sabido es que el Teatro Cervantes era de grandes dimensiones, dominando toda una manzana en la Plaza del Campillo, y que durante muchos años parte de los bajos de este inmueble fue ocupada por el Liceo Granadino. 
El Liceo Artístico y Literario Granadino, en que con motivo de la coronación del egregio Zorrilla hubo de improvisar el primer salón del palacio de cristal que se adhiere al vetusto teatro como la hiedra al álamo para que le sirva de sostén: ambos viven unidos perfectamente y en él ha dado ya la culta sociedad liceísta alguno de sus mejores recreos en estos últimos tiempos. Son dos buenos amigos de antigua y gloriosa historia, que por diversos caminos contribuyen al esplendor de las letras y las artes granadinas y que marchan abrazados por la senda que le trazaron sus ilustres progenitores.

El 22 de enero de 1966, un grupo de aficionados granadinos, junto con actores profesionales y veteranos de la escena y la radio, dirigidos por D. Manuel Hernández, pusieron en escena La Venganza de D. Mendo. La función consistió en un doble homenaje: por un lado, al Teatro Cervantes, que iba a desaparecer definitivamente de la vida de la ciudad para dar paso a un bloque de pisos y bajos comerciales, y en segundo lugar a D. Manuel Hernández Bermúdez, que durante muchos años había sido animador de empresas escénicas en Granada.

Ocupando un lugar predilecto en la ciudad de Granada y sobre los escombros del Teatro Cervantes, en la actualidad encontramos un inmueble intrascendente, que poco o nada añade al conjunto histórico de una ciudad especialmente sensibilizada con el arte.

\section{Teatro Isabel la Católica}

El 6 de junio de 1952 se inauguraba un nuevo teatro en Granada, que no era otro que el que hoy en día podemos ver en la Acera del Casino. Surgió en el lugar donde estuvo emplazado el Casino Principal que, tras su trágico incendio, desapareció, quedando el solar ocupado durante los años de la posguerra por el cine de verano "Palermo". Este nuevo teatro fue bautizado con el nombre de Isabel la Católica, posiblemente en recuerdo del que existiera en la Plaza de los Campos, incendiado también en I936. Su arquitecto fue Miguel Olmedo Collantes.

En función de gala y con la ópera de Bizet 'Carmen', quedó anoche inaugurado el gran teatro Isabel la Católica en que se dio cita la aristocracia granadina, que llenó todas las localidades 
del suntuoso coliseo. Las damas vestían trajes de noche y los caballeros de etiqueta, pincelada que abrillantó el estreno de la sala, de gran vistosidad y riqueza en mobiliario y apliques y con muy artística iluminación.

Al igual que en otros actos de inauguración, fue la Asociación de la Prensa la patrocinadora de su apertura. Un numeroso público se congregó en los alrededores del nuevo teatro para presenciar la llegada de las autoridades invitadas, entre los que se encontraban igualmente la famosa artista Joan Fontaine, el galán Louis Jourdan y el cineasta Hugo Fregonese, que se hallaban rodando una película en Granada.

Carmen de Bizet, fue la ópera elegida para la inauguración. Del gran éxito que alcanzó esta representación, hay que subrayar a Leoz por su voz pastosa y dramática, y a Chano Gonzalo, como también a la que es gran cantante y actriz Tony Rosado. Ante los insistentes aplausos ‘bisaron' el dúo del segundo acto.

Según indica Juan Bustos en su obra Viaje al centro de Granada, los abonos, que se agotaron rápidamente, fueron los siguientes: "Butacas de patio, para las tres funciones, noventa pesetas; palcos con seis entradas, trescientas sesenta; butacas de club, sesenta y cinco pesetas; butacas de principal, treinta y cinco".

El teatro fue muy alabado por el público en general, siendo muy del gusto de los asistentes las pinturas al temple del zaguán y la cúpula de la sala, realizadas por un artista granadino llamado Manuel Rivera: "inspiradas en obras de nuestro teatro, como D. Juan Tenorio, Doña Rosita la soltera (...), y en cuyos personajes Manolo Rivera había reproducido, con notable acierto, las caras de algunos de sus mejores amigos: Manuel Gallego Morell, Antonio Moscoso, Joaquín Serrano, Manuel Maldonado. En las figuras femeninas, el rostro es el de María Luisa Navarro, entonces novia del pintor”. Sería D. Antonio Gallego quien le sugiriera los temas.

Según relataba Juan Bustos en el periódico Ideal, la primera gran aglomeración de público a las puertas de este nuevo teatro se produjo a los pocos días de su inauguración: un atrevido paracaidista (que se anunciaba con el nombre de Jans Hill), decidió tirarse desde la azotea 
del Teatro Isabel la Católica. Realizó su salto, consiguiendo que se abriera su paracaídas poco antes de rozar las copas de los árboles de la Acera del Casino.

Después de haber pertenecido durante largo tiempo a la Organización Española Mercantil S. A. Granada, el Teatro Isabel la Católica fue adquirido por el Ayuntamiento. Sin embargo, ante el penoso estado de conservación en que se encontraba, el alcalde de Granada ordenó su clausura el 2I de julio de I992. "Quero mantendrá próximamente una reunión con el director general de arquitectura de la Junta para tratar las obras de rehabilitación integral que necesita el edificio. Todos los espectáculos han sido suspendidos hasta que no finalicen los trabajos de remodelación" (s/p).

El cierre del teatro obligó a suspender todas las actuaciones y representaciones previstas, afectando principalmente a las del Festival de Jazz y a las del Festival Internacional de Teatro.

Casi seis años después de que se bajara el telón, el Teatro Isabel la Católica abre de nuevo sus puertas con la anunciada presencia de la ministra de cultura Esperanza Aguirre. Esto ocurría el 26 de mayo de 1998. Tras una remodelación en la que se invirtieron más de 700 millones de pesetas y superar infinidad de incidencias que fueron retrasando las obras, el teatro cobraba vida nuevamente con una obra de Federico García Lorca: Mariana Pineda.

\section{España: un país para la inclusión}

Hace mas de treinta años que España comenzó su andadura hacia la inclusión educativa: los niños con discapacidad educativa deberían a partir de entonces integrarse en las aulas de los centros públicos y concertados. Para ello era indispensable la consiguiente formación específica del profesorado que incidiría, directamente, en la atención "especial" que requiere este alumnado, en cuanto a los objetivos pedagógicos y quizá, los más importantes, los personales.

Debemos matizar aquí que, si bien los primeros pasos se dieron hace tres décadas, este camino no se ha recorrido con toda la velocidad que hubiera sido deseable. Los centros escolares se muestran en algunos 
casos reacios a profundizar en este campo, ya que en muchas ocasiones se confunde la "inclusión" con la "integración", siendo esta última el hecho de que alumnos con "otras capacidades" convivan en el aula con el resto del alumnado, mientras que la primera requiere de una formación y cambio de la metodología habitual, que no siempre son bien recibidos por los centros educativos.

Asimismo, a la hora de abordar el tema de la inclusión, suele ser también más común y más fácil de percibir la necesidad de adaptar las medidas pedagógicas cuando el alumnado presenta una discapacidad física a cuando el alumnado presenta necesidades de tipo social. En este último caso no solo es imprescindible un apoyo gubernamental (leyes, recursos económicos, dotación de personal especializado...), sino que el pesquis del docente en cuestión sea el encargado de buscar los recursos que tenga a la mano para "enganchar" al alumnado con necesidades y al resto sus compañeros en su andadura con ellos.

\section{Educamos en la igualdad}

El idioma español es prolijo en sinonimia y polisemia, y los españoles aprovechamos bien esta cualidad de nuestra lengua. Uno de los términos "de moda" es el de "igualdad", si bien en nuestro caso lo vamos a tomar orientado hacia la voluntad de hacer de nuestros alumnos personas con igualdad de oportunidades. Pero, aunque sin profundizar en la idea, esto que se presenta como algo obvio es en realidad una tarea mucho más compleja de lo que puede parecer. Los recursos educativos que pone a disposición el Estado y de los que disfrutan los centros educativos son los mismos, pero ¿todos los alumnos realmente "disponen de ellos"? ¿Todos los alumnos los van a utilizar? Y aquí la respuesta rápida que se nos viene a la cabeza es: "pues los utilizará el que quiera, o el que los necesite", y olvidaríamos quizá que gran parte del alumnado no puede utilizar diversos recursos porque no los conoce. Y no me estoy refiriendo a casos en que los profesores los empleen de tal o cual manera, me estoy refiriendo al alumno como ente individual que, en ciertos casos, tiene una serie de carencias que "le impiden ver el bosque tras los árboles". 
Es función primordial del personal de los centros educativos prender (o por lo menos intentar) la mecha de la curiosidad en esos alumnos que, por las circunstancias que sea, no están en "igualdad" de condiciones que sus compañeros. Y en este punto hay dos elementos fundamentales que ayudan a esta tarea: utilizar los recursos de que se dispone, más allá de las fronteras del centro escolar, y poner al alumno en "situación de éxito", porque no hay nada más satisfactorio para el ser humano que sentirse líder, ver que puede tanto o más que el resto de sus compañeros. Y en líneas anteriores dijimos "o por lo menos intentar", y aquí debo hacer un inciso: hay que intentar, no parar de intentar, porque lo que hoy no te sirve con un alumno, sí te puede servir para otro, pero entonces tendrás que seguir intentando motivar e igualar al primero, y tendrás que buscar cómo, y aunque muchas veces parece que es eso — solo un intento-, en realidad vas a poder "incluir" a alumnos que, de otro modo, seguirían sin sentirse ni iguales, ni incluidos.

\section{La "innovación educativa", un plan para todos}

"Innovación pedagógica". Dos palabras que se han convertido en meta oficial y extraoficial de gran número de centros educativos. Oficial porque, al fin y al cabo, los centros educativos compiten por atraer al mayor número posible de alumnado y poder mantener así sus puertas abiertas. Extraoficial porque es en realidad un conjunto de actuaciones que funcionan y mejoran el centro a la hora de hablar de igualdad, de integración y, como no, de inclusión.

La innovación pedagógica no es más (ni menos, porque en sí la idea es fácil pero el camino es arduo) que cambiar el "cómo" para mejorar el "qué". Es implantar una serie de actuaciones, procesos, herramientas, estrategias... que van a cambiar el papel de todos los elementos de la comunidad educativa y mejorar el desarrollo de las competencias e "inteligencias" del alumnado.

En este aspecto, son muchos los caminos que pueden escogerse. De hecho, ni siquiera hay que escoger un solo camino y podrían combinarse entre sí. "Aprendizaje Basado en Proyectos"; "Aprendizaje Basado en Problemas", o "Aprendizaje Cooperativo" son algunas de las nuevas 
metodologías que pretenden estimular al alumnado y que son mucho más eficaces que las metodologías tradicionales a la hora de tratar con alumnado en riesgo de exclusión. Este tipo de enseñanza, como anticipamos, no varía los contenidos, que son los marcados por la ley según cada Comunidad Autónoma. Con ella, los alumnos participan de forma activa, todos, ya que cada uno aporta lo que sabe o lo que puede dentro de la tarea correspondiente, y además eso que aporta es indispensable para lo que tienen que aportar los demás, con lo que no hay pérdida posible. Todos estarán en "situación de éxito" con el resto de sus compañeros, mejorará las relaciones interpersonales entre ellos, potenciarán sus capacidades y, además, adquirirán los contenidos de la materia en cuestión. No hay desperdicio.

\section{La Manigua nos lo pone fácil}

"Manigua” es un término de origen taíno, sustantivo que se refiere a "copiosidad, abundancia, exuberancia, acopio o demasía de manera desordenada de algo, confusión, consternación o desconcierto, cuestión intrincado”. Este fue el nombre escogido para un barrio en el que se practicaban actividades "desordenadas", que se abandonaron tras la remodelación de Gallego Burín y que ahora es un itinerario de enorme belleza en pleno centro de la capital granadina. La confluencia entre tradición y modernidad en este espacio hace que sea uno de los más atractivos de la ciudad, no solo para aquellos que se recrean en lo histórico y cultural, sino también para los que buscan un momento de esparcimiento. Esta conjunción lo convierte en un itinerario perfecto para la consecución de la tan perseguida "inclusión".

¿Cuáles son los elementos atractivos en este itinerario para el alumnado en riesgo de exclusión social o con cualquier otra "discapacidad"? Me encantaría poder decir que los alumnos van a llegar a este barrio interesados por su historia, por su riqueza arquitectónica y patrimonial, o por sus teatros. Claramente, no es así. Pero, por otro lado, ¿qué papel tendríamos los docentes si nuestros alumnos reunieran ya a priori esas inquietudes? Poco nos quedaría por hacer. No obstante, a la hora de hablar de itinerarios, todos van a tener en común un hecho que los hace en sí mismo atractivos: están en la calle, hay que salir de los muros de "la escuela", hay que 
pasear, mirar, oír, les da el aire, cambian la rutina escolar y eso es, en sí mismo, un poderoso atractivo.

Y partiendo de esa idea de "aire libre", plantear un itinerario en el que descubramos la historia del Barrio de la Manigua pasa por estudiar la Edad Media, la Edad Moderna, la realidad social de los lupanares de antes y de ahora, el franquismo, el urbanismo, la tradición vs. la modernidad, los teatros como lugar de esparcimiento, los teatros como lugar en el que todos tenemos cabida... La lista de temas con los que entroncar este itinerario con el alumnado es infinita, pues las relaciones entre ambos las van a establecer de forma individual los propios alumnos, aunque guiados por el docente que las plantee. Es la mejor manera de preservar la historia y hacer verdadera inclusión.

\section{Una metodología emocional}

Debemos dejar claro que nuestra labor como docentes será la de guiar y coordinar la actividad, ejerciendo un aprendizaje cooperativo basado en el desarrollo de grupos. En estos grupos va a haber un aumento de la interacción entre los miembros, siendo estas interacciones diferentes debido a las sensibilidades de nuestro alumnado.

Esta acción va a dar garantizar que todos interactúen en esos pequeños grupos, favoreciendo la autoestima y mejorando el sentido de la autoeficacia, ya que entre ellos se van a ayudar más, realizando asimismo un ejercicio de construcción de la solidaridad y, por añadidura, se dará lugar a una situación en la que el éxito vendrá del ejercicio personal del equipo. Por lo tanto, tendrán que trabajar de igual manera para cumplir el objetivo básico de este Itinerario cultural, que radica en el descubrimiento, explicación y disfrute del Barrio de La Manigua desde la perspectiva de la inclusión y el patrimonio histórico artístico, y apoyados en las TIC.

\section{Plan de trabajo y actividades a realizar}

El plan de trabajo radica en primer lugar en establecer grupos de trabajo con el fin de llevar a cabo este itinerario cultural. Desarrollaríamos el Itinerario en unas 5 sesiones: 
- Sesión l: se basaría en la presentación de este proyecto/itinerario cultural, que titularíamos El Barrio de la Manigua: conocer su pasado, contemplar en el presente y preservar para el futuro, en el que crearíamos 4 grupos de trabajo, con el fin de distribuir cada grupo a un área de las expuestas anteriormente (Plaza del Carmen, Puerta Real, Ángel Ganivet y Plaza de Mariana Pineda), para que investigue ese espacio. En esta sesión se haría una lluvia de ideas previas sobre los conocimientos que posee el alumnado sobre esta zona (si es que los tiene) y, tras esto, se les entregaría un mapa en papel para delimitar el espacio de estudio y específicamente el que a cada grupo le ha tocado trabajar. Posteriormente se procedería a una explicación general de la historia de nuestro itinerario cultural, específicamente el contexto histórico-artístico general y la explicación de la importancia de valorar el patrimonio local del Barrio de La Manigua.

- Sesiones 2 y 3: se procedería a la entrega de los cuestionarios que, a través de las TIC, bibliotecas y de la consulta con personas del Barrio de la Manigua, podrán rellenar para, en la sesión posterior, utilizar en la explicación del desarrollo del itinerario. Las preguntas modelo a las que deberían de responder usando las TIC (Internet, Google Maps, Youtube...) serían las siguientes:

¿Qué localización tiene en el mapa el espacio que se te ha asignado para investigar?

¿Dónde se ubica?

¿Qué edificios existen?

¿Quién lo construyó y en qué fecha?

¿Cómo se llaman los hombres o mujeres que lo construyeron? ¿Porqué se construyó? ¿Con qué finalidades?

¿A qué se debe su nombre?

¿Qué usos tuvo en el pasado?

¿Qué usos tiene en el presente?

¿Hay alguna fotografía del pasado que puedas encontrar en Google Imágenes? 
¿Hay algún testimonio de alguna persona que haya visto la transformación y evolución de este espacio?

¿Hay alguna publicación o libro donde se hable del espacio que vas a investigar?

¿Hay algún video en Youtube en el que se explique?

- Sesión 4: llevaríamos al alumnado integrante de los diferentes grupos a los espacios que se les ha asignado investigar, para que expliquen con sus palabras lo aprendido e investigado, para que sean ellos y ellas los verdaderos protagonistas de este aprendizaje y cuenten los problemas existentes o no, de si han encontrado datos, si han sido efectivos. Por ejemplo, se contrasten las fotografías del pasado con la realidad de los espacios, etc.

- Sesión 5, la puesta en común: quizás ésta sea la sesión más importante de todo el Itinerario junto con la anterior (4). Una vez visitado, explicado y contemplado el patrimonio del Barrio de La Manigua se realizará una puesta en común en el aula, organizado el alumnado en sillas de forma circular, para que cada uno exponga, de forma oral, al resto de sus compañeros las cuestiones positivas y negativas que han desarrollado en ese trabajo en común —en primer lugar en los grupos de trabajo establecidos-, para, después, valorar a sus compañeros en referencia a su exposición en la sesión previa, fortaleciendo y aprendiendo de una forma conjunta.

\section{Conclusión}

El resultado de este itinerario permitirá mirar con una sensibilidad especial este barrio olvidado de los estudios patrimoniales, focalizar su atención por parte del estudiantado, ya que son en definitiva el futuro de la sociedad. A la vez, romper con la cotidianeidad del aula y abstraerse en este barrio fuera del aula, aplicando su propio aprendizaje y experiencia, y creando en la memoria un recuerdo imborrable de ese itinerario cultural.

La práctica de este Itinerario permitirá, desde la diversidad del alumnado, aplicar sus singularidades en el aprendizaje y conocimiento, para comprender, de esta manera, que la enseñanza de la historia y el arte no está ceñida únicamente a la lección magistral del profesorado, 
sino que ellos, hoy más que nunca, forman y deben de formar parte del proceso de enseñanza y aprendizaje del conocimiento de la historia granadina.

\section{Referencias bibliográficas}

Álvarez E., Álvarez M., Castro O., Campo A. y Fueyo E. (2008). Funcionamiento de la integración en la Enseñanza Secundaria Obligatoria según la percepción del profesorado. Psicotbema, 20, 56-62.

Bustos, J. (1996). Viaje al centro de Granada. Granada: Ediciones Albaida.

Contreras García, J. (2017). Granada (1936-1956) Dos décadas de creatividad arquitectónica en el primer franquismo. Un estudio desde la perspectiva didáctica. Granada: Universidad.

Echeita G. y Verdugo M. A. (2005). Diez años después de la Declaración de Salamanca sobre Necesidades Educativas Especiales en España. Entre la retórica esperanzadora y las resistencias al cambio. Siglo Cero, 36, 5-I2.

Extremera, N. y Fernández-Berrocal, P. (2004). El papel de la inteligencia emocional en el alumnado: evidencias empíricas. Revista Electrónica de Investigación Educativa, 6(2).

Gallego Burín, A. (1982). Granada: guía artística e bistórica de la ciudad. Granada: Don Quijote.

García-Pastor C. (2003). Segregación, integración e inclusión. Bordón, 55, 9-26.

Isac, A. (2007) Historia urbana de Granada: formación y desarrollo de la cindad burguesa. Granada: Diputación.

Juste, J. (1995). La Granada de Gallego y Burin, 1938 195l. Reformas urbanas y arquitectura. Granada: Diputación.

Orduz, R. (dir). (2012). Tecnologias de la información para la inclusión social: una apuesta por la diversidad. Universidad del Norte. Corporación Colombia Digital, Bogota D.C., Colombia Octubre 2012. Recuperado de https:// colombiadigital.net/herramientas/nuestras-publicaciones/sociedad-ycalidad-de-vida/item/3938-tic-para-la-inclusion-social-una-apuestapor-la-diversidad.html Consulta: 28/12/2018.

Ortiz M. C. y Lobato X. (2003). Escuela inclusiva y cultura escolar: algunas evidencias empíricas, en C. García Pastor y M. C. Ortiz González (coords.), Más allá de la Educación Especial, Monográfico, Bordón, 55, 27-40.

Vallés Arándiga, A. (2016). Psicopedagogía de la inteligencia emocional. Formación continuada Logoss. 
Verdugo Alonso M. A. y Rodríguez Aguilella A. (2012). La inclusión educativa en España desde la perspectiva de alumnos con discapacidad intelectual, de familias y de profesionales. Revista de Educación, 358, 450-470.

\section{Hemeroteca}

B, Juan. “Teatro Isabel la Católica”. Ideal, Granada, 6 de noviembre de 2000, p. 8.

“Correos estrena Casa” Ideal. Sección "te recuerdo” 26 de junio de 2013.

“Crónicas Granadinas”. Revista Alhambra. tomo VI, Granada: I903, pp. 2I5-2I6.

"El Ayuntamiento clausura el Isabel la Católica y revela la inseguridad en el teatro desde hace años”. Ideal, Granada, 22 de julio de I992, n I9.0I6, p. 4.

"Granada recupera hoy el Teatro Isabel la Católica, tras seis años de obras". Ideal, Granada, 26 de mayo de I998, n² 2I.I24, p. II.

"Junta de Espectáculos”. El Defensor, Granada, I de diciembre de 1912, n I6.I46, p. I.

"Las primeras autoridades y la alta sociedad, en la inauguración del Teatro Isabel la Católica”. Ideal Granada, 7 de junio de I952, n 6.I58, p. 2.

Miguel Olmedo Collantes. "Proyecto de nueva fachada para el Hotel Victoria". Ideal, 6 de mayo de 1943, p. 5.

Millán Ferriz, E. "El Teatro Principal”. El Defensor, Granada, 25 de febrero de I894, nº 6.632, p. 2.

Consultas de archivo

Construir en la calle Ángel Ganivet. Archivo Histórico Municipal de Granada, signatura, C.03080.0865 (I943).

Miguel Olmedo Collantes. Sobre pavimentación de la calle "A" en la zona baja de la Manigua. Archivo Histórico Municipal. 